\title{
A Probabilistic Framework for Highway Safety Analysis ${ }^{1}$
}

\author{
Jianghai Hu, John Lygeros, Maria Prandini and Shankar Sastry \\ Department of Electrical Engineering and Computer Sciences \\ University of California, Berkeley CA 94720 \\ $\{j$ ianghai, lygeros, prandini, sastry\} Orobotics.eecs.berkeley.edu
}

\begin{abstract}
Brownian motion is used to model the uncertainty in the motion of cars on a highway. The probability of collision of two adjacent cars within a fixed horizon is calculated and its implications are discussed. Moreover, the probability of collision in the presence of emergency braking is also obtained by modeling the occurrence of emergency braking as a Poisson process.
\end{abstract}

\section{Introduction}

Consider the following first order stochastic differential equation:

$$
\frac{d x}{d t}=f(t)+w(t)
$$

where $f$ is a continuous function defined on $[0, \infty)$ and $w(t)$ is white noise with power spectral density $\sigma^{2}$, i.e., $E[w(t) w(t+s)]=\sigma^{2} \delta(s)$. Integrating equation (1), we have

$$
x(t)=\int_{0}^{t} f(s) d s+b(t)
$$

where $b(t) \triangleq \int_{0}^{t} w(s) d s$ is a Gaussian process with stationary, independent increment. Furthermore $E[b(t)]=$ $\int_{0}^{t} E[w(s)] d s=0$ and

$$
\operatorname{Var}[b(t)]=\int_{0}^{t} \int_{0}^{t} E\left[w\left(s_{1}\right) w\left(s_{2}\right)\right] d s_{1} d s_{2}=\sigma^{2} t
$$

These properties together with the assumption that $b(t)$ is continuous in $t$ imply that, after scaling by $1 / \sigma, b(t)$ is a standard Brownian Motion (BM). Although the continuous path assumption is not a logical consequence of the previous hypotheses, (2) and the Kolmogorov continuity theorem imply that $b(t)$ has a continuous version $B_{t}$, i.e., $P\left(\left\{\omega: b(t, \omega)=B_{t}(\omega)\right\}\right)=1$ for all $t \geq 0$ and $\left\{B_{t}(\omega): t \geq 0\right\}$ is continuous in $t$ with probability 1 (see [1]). The continuity assumption is further justified when equation (1) is used to model the

\footnotetext{
${ }^{1}$ Research supported by the California PATH program under MOU-312, MOU-319, by DARPA under grant F33615-98-C-3614 and by ARO under MURI DAAHO4-96-1-0341.
}

motion of physical objects, since in this case $x(t)$ cannot make instantaneous jumps.

In this paper we apply the solution to equation (1) to a 1-dimensional road model, in which a car (possibly in a platoon [2]) needs to predict the motion of adjacent cars to determine a safe distance from them. The prediction is based on the assumption (belief) that adjacent cars will try to maintain their current speeds, but may not be able to do it precisely, due to factors such as road conditions, wind, mechanical malfunction, and so on.

The use of BM for modeling uncertainty in motion can be justified by the fact that BM can be thought of as the accumulation of a large number of independent small disturbances. More precisely, subdividing time into intervals of length $\Delta t$, assuming that the disturbances in different time intervals are independent and identically distributed (IID), and integrating, we get a random walk which, after appropriate scaling, converges in distribution to a standard BM as $\Delta t \rightarrow 0$ (see [3]). For example, in [4] it is verified by empirical data that the prediction error for a cruising aircraft is indeed Gaussian with a growing variance. Another important application of equation ( 1 ) to air traffic management is in fact discussed in a separate paper [5].

It should be noted that BM possesses many unusual local properties; for example, at any time it is not differentiable with probability 1 . However, here we are mainly concerned with its collective properties, i.e., the probability that the perturbed trajectory experiences a large deviation from the nominal one or, equivalently, the state $x$ evolves outside some subset of the state space called the safe set. For the road model the safe set can be simply defined as the set of states where no part of any pair of cars occupies the same physical space at the same time. By subtracting the nominal motion $\int_{0}^{t} f(s) d s$ and properly scaling, we can adopt an alternative view point where the perturbed motion consists only of a standard $\mathrm{BM},\left\{B_{t}, t \geq 0\right\}$, and the safe set is time-varying. In this new coordinate system, the problem reduces to calculating the hitting probability of the standard BM with respect to a time-varying region.

Remark: In the case of time-invariant safe set, a deep connection exists between excursion of BM and classical 
potential theory, which can be used to calculate the exit distribution of a $\mathrm{BM}$ with respect to certain regions (see [6]). However, here we are more interested in the exit time distribution from a time-varying safe set.

The paper is organized in five sections. In Section 2 an expression for the probability of collision (PC) without emergency braking is obtained, and its implications on the spacing of vehicles in a platoon are briefly discussed. Then, the presence of emergency braking is considered and the notion of deterministic safe distance is introduced in Section 3. An expression for $\mathrm{PC}$ with emergency braking is derived in Section 4, and finally concluding remarks and directions for future work are given in Section 5. The Appendix contains some additional formulae used in the derivations.

\section{PC without emergency braking}

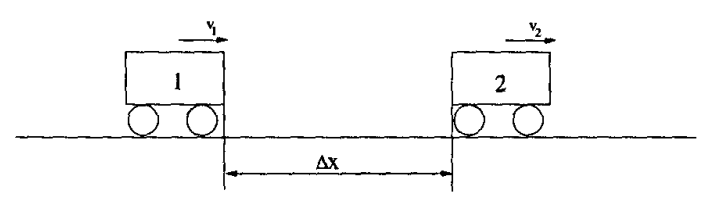

Figure 1: 1-dimensional highway model.

Consider two adjacent cars, labeled 1 and 2, with car 2 in the lead, moving at speed $v_{1}$ and $v_{2}$ respectively down a highway (Figure 1). Denote with $\Delta x$ their initial distance and with $\Delta v \triangleq v_{2}-v_{1}$ their velocity difference. We use equation (1) to model the uncertainty in the motion of the two cars due to measurement errors and various unknown environment factors, and derive the probability of collision (PC). For the time being, we ignore the possibility of emergency braking. The computation of $\mathrm{PC}$ in the presence of emergency braking is postponed to Section 4 .

The motion of car 2 is modeled as a deterministic motion at constant velocity $v_{2}$ plus a scaled BM perturbation $\sigma B_{\mathrm{t}}$ for some $\sigma>0$, whereas car 1 is assumed to be moving deterministically at constant velocity $v_{1}$. Although one may argue that the motion of car 1 should also be stochastic, we can simply attribute the perturbation in the motion of car 1 to that of car 2 and get a combined scaled BM perturbation, provided the two perturbations are independent. Scaling $\Delta x$ and $\Delta v=v_{2}-v_{1}$ by a factor of $1 / \sigma$, we can further assume that the perturbation is a standard 1-D BM $B_{t}$ with $\operatorname{Var}\left[B_{t}\right]=t$. The new initial distance and velocity difference of the two cars will then become:

$$
\Delta x^{\prime} \triangleq \Delta x / \sigma, \quad \Delta v^{\prime} \triangleq \Delta v / \sigma .
$$

The degree of danger of a particular situation is defined as the probability of collision within a fixed time $t_{f}$ (it does not make much sense to consider the danger of

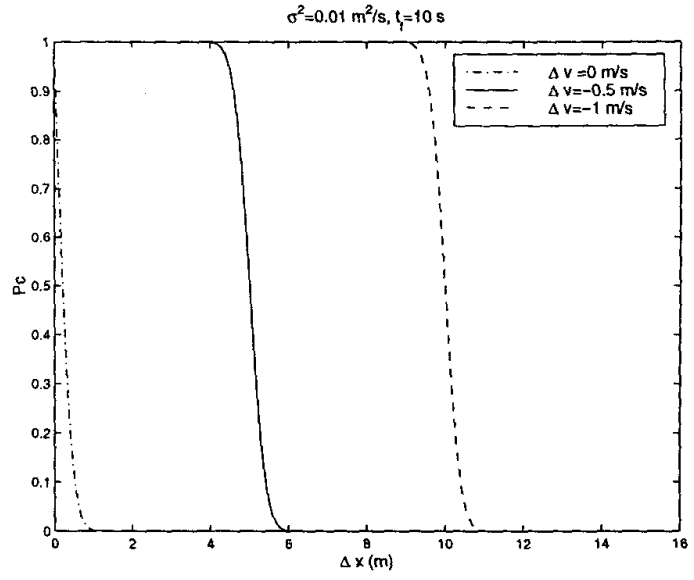

Figure 2: Hitting probability with $t_{f}=10 \mathrm{~s}$.

two cars hitting after two days). Notice that although car 1 can choose to brake once the distance $\Delta x^{\prime}$ becomes too small, we assume car 1 continues to move at its current velocity in the near future, because danger is by our definition the possibility that collision occurs within some fixed time if the current settings remain unchanged.

We can adopt the equivalent viewpoint that car 2 is at the fixed position $x=\Delta x^{\prime}$ and the motion of car 1 is the combination of a deterministic motion with constant velocity $\mu=-\Delta v^{\prime}=\left(v_{1}-v_{2}\right) / \sigma$ and a $1-\mathrm{D}$ standard BM $B_{t}$, i.e., a Brownian motion $\left\{B_{t}^{\mu}, t \geq 0\right\}$ starting from 0 and with drift term $\mu\left(B_{t}^{\mu}=B_{t}+\mu t\right)$. In this setting, a collision can be viewed as the event that $\left\{B_{t}^{\mu}, t \geq 0\right\}$ reaches $\Delta x^{\prime}$. Hence the problem becomes calculating the probability that this event occurs within time $t_{f}$. Define $T \triangleq \inf \left\{t \geq 0: B_{t}^{\mu}=\Delta x^{\prime}\right\}$ to be the first time $B_{t}^{\mu}$ reaches $\Delta \boldsymbol{x}^{\prime}$.

Lemma 1 (Bachelier-Levy, [3]) $T$ has probability density

$$
p(t)=\frac{\Delta x^{\prime}}{\sqrt{2 \pi t^{3}}} \exp \left(-\frac{\left(\Delta x^{\prime}-\mu t\right)^{2}}{2 t}\right) .
$$

Denote with $A$ the event that a collision occurs before time $t_{f}$, i.e., $A \triangleq\left\{B_{t}^{\mu} \geq \Delta x^{\prime}\right.$, for some $\left.t \in\left[0, t_{f}\right]\right\}$. Since $A=\left\{T \leq t_{f}\right\}$, it is not hard to get from Lemma 1 and Formula 2 in the Appendix that:

$$
P(A)=Q\left(\frac{\Delta x^{\prime}-\mu t_{f}}{\sqrt{t_{f}}}\right)+e^{2 \mu \Delta x^{\prime}} Q\left(\frac{\Delta x^{\prime}+\mu t_{f}}{\sqrt{t_{f}}}\right),
$$

where $Q(z) \triangleq \int_{z}^{\infty} \frac{1}{\sqrt{2 \pi}} \exp \left(-t^{2} / 2\right) d t$. Using equation (3), Theorem 1 then immediately follows. 
Theorem 1 The probability of collision within time $t_{f}$ without emergency braking, $P_{c}(\Delta v, \Delta x)$, is:

$$
Q\left(\frac{\Delta x+\Delta v t_{f}}{\sigma \sqrt{t_{f}}}\right)+e^{-2 \Delta x \Delta v / \sigma^{2}} Q\left(\frac{\Delta x-\Delta v t_{f}}{\sigma \sqrt{t_{f}}}\right) .
$$

Figure 2 shows some plots of $P_{c}(\Delta v, \Delta x)$ as a function of $\Delta x$ for different values of $\Delta v$, with $t_{f}=10 \mathrm{~s}$ and $\sigma^{2}=0.01 \mathrm{~m}^{2} / \mathrm{s}$. Note that $P_{c}$ decreases from 1 to 0 at around $\Delta x=-\Delta v t_{f}$. So if we specify the safe distance by requiring $P_{c}$ to be below some threshold, then it will be a decreasing function of $\Delta v$. In addition, if a larger $\sigma$ is chosen, the transition of $P_{c}$ from 1 to 0 will be less abrupt.

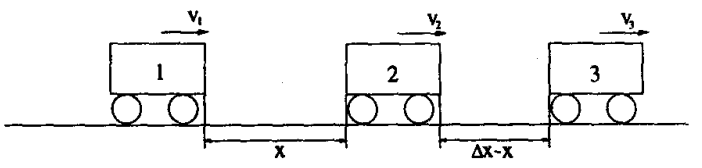

Figure 3: A three car platoon.

To further test the qualification of $P_{c}$ to be a measure of degree of danger, let us consider the situation of a three car platoon (Figure 3 ). Denote with $\Delta x$ the initial distance between car 1 (last) and car 3 (first), excluding the length of car 2 , and with $\Delta v$ their velocity difference, $\Delta v \triangleq v_{3}-v_{1}$. We try to determine what are the safest velocity $v_{2}$ and initial distance $x$ from car 1 of car 2 , in the sense that

$$
\begin{aligned}
D(v, x) & =P_{c}\left(v_{2}-v_{1}, x\right)+P_{c}\left(v_{3}-v_{2}, \Delta x-x\right) \\
& =P_{c}(v, x)+P_{c}(\Delta v-v, \Delta x-x)
\end{aligned}
$$

is minimized, where we set $v \triangleq v_{2}-v_{1}$. Note that this criterion is not the probability of car 2 hitting either car 1 or car 3 before time $t_{f}$.

A straightforward calculation shows that $v_{0}=\Delta v / 2$, $x_{0}=\Delta x / 2$ is a solution to the equations:

$$
\frac{\partial D}{\partial v}=0 \quad \frac{\partial D}{\partial x}=0
$$

Furthermore it can be verified that $\left(v_{0}, x_{0}\right)$ is the global minimum of $D(v, x)$ on $R \times[0, \Delta x]$ provided $\Delta x+\Delta v \cdot t_{f} \gg 0$; if this is not the case, car 1 and car 3 will collide by time $t_{f}$ with a probability close to 1 , so there is nothing car 2 can do to avoid a collision with one of them. The obtained result agrees with our intuition since it implies that the safest position and velocity for car 2 is the average of those of car 1 and car 3. In [7] it is shown by means of simulations that controllers implementing this intuitive arrangement are better suited for emergency maneuvers for platoons.

Figure 4 shows a plot of $D(v, x)$ together with some of its level sets. We deliberately chose a large $\sigma^{2}, \sigma^{2}=$

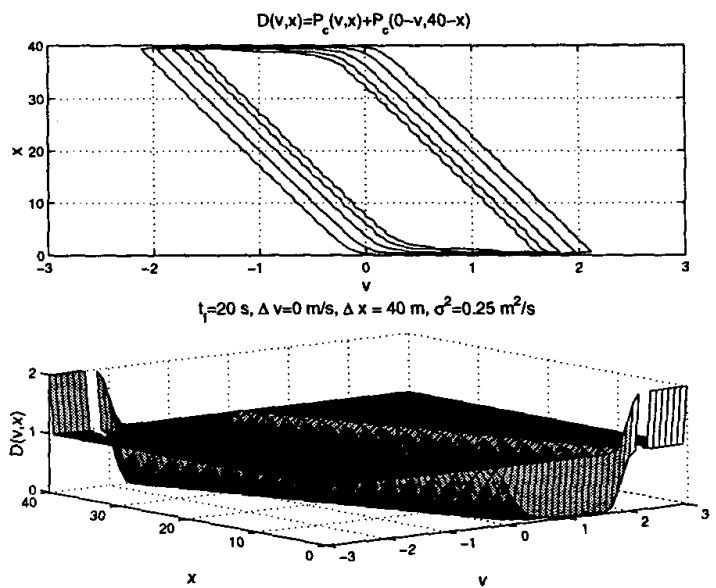

Figure 4: Overall hitting probability.

$0.25 \mathrm{~m}^{2} / \mathrm{s}$, so that $P_{c}$ can be calculated for a wider range of $x$ and $v$ without numerical overflow. Notice that if we define $v_{0}(x)$ as the optimal velocity difference obtained by minimizing $D(v, x)$ over $v$ for fixed $x$, then $v_{0}(x)$ is nearly linear as a function of $x$. This fact can assist in the design of controller for cars in a platoon.

\section{Safe distance under emergency braking}

Consider the scenario in Figure 1. Assume for the time being that there is no uncertainty in the motion of the two cars and their maximal deceleration capabilities are $-a_{1}$ and $-a_{2}$ respectively, with $a_{1}, a_{2}>0$. Suppose that, at some time instant $t$, car 2 brakes as hard as it can, due to some unexpected reason (e.g. failure, obstacle, road accident ahead, etc.). The question is what is the minimal distance $d_{\min }$ between the two cars at time $t$ such that no collision will occur.

Without loss of generality, we set $t=0$. Suppose car 1 responds by braking as hard as it can without any delay. Then car 1 will travel a distance of $S_{1}=v_{1}^{2} / 2 a_{1}$ before stopping at time $t_{1}=v_{1} / a_{1}$. Similarly for car 2 , $S_{2}=v_{2}^{2} / 2 a_{2}$ and $t_{2}=v_{2} / a_{2}$. A necessary condition of no collision is $S_{2}+\Delta x>S_{1}$, i.e.,

$$
\frac{v_{2}^{2}}{2 a_{2}}+\Delta x>\frac{v_{1}^{2}}{2 a_{1}} \text {. }
$$

A typical plot of the positions of two cars as functions of time during an emergency braking process is shown in Figure 5. Assume $\Delta x$ satisfies condition (4). To derive the necessary and sufficient condition so that no collision will occur, we distinguish the following cases:

Case 1: $a_{1} \leq a_{2}$. No collision can occur in this case because $a_{1} \leq a_{2}$ implies that the curvature of the parabola describing the position in time of car 2 is greater than 


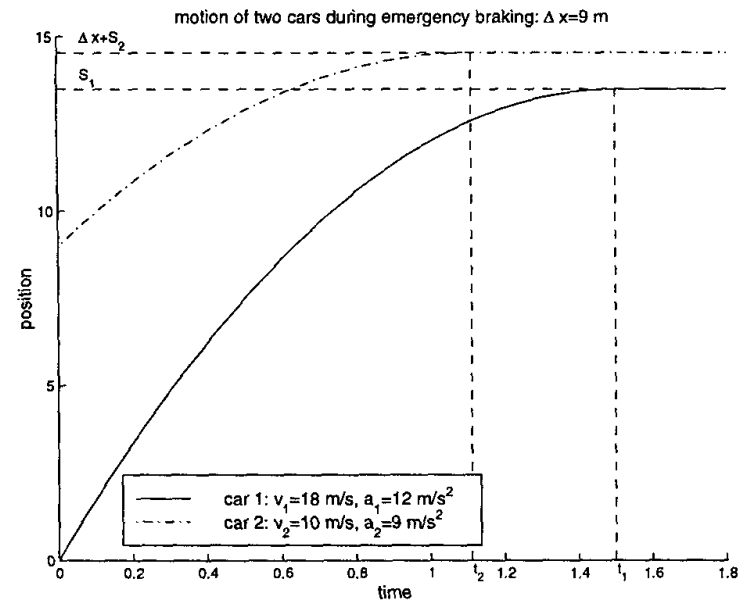

Figure 5: Typical motions of two cars during emergency braking.

(if $a_{1}<a_{2}$ ) or equal to (if $a_{1}=a_{2}$ ) that of car 1 , so there is no collision up to time $\min \left\{t_{1}, t_{2}\right\}$ which in turn implies no collision up to time $t_{1}$ when car 1 stops.

Case 2: $a_{1}>a_{2}$. We distinguish two sub-cases:

1. If $t_{1} \geq t_{2}$, then no collision occurs as one can easily see by shifting the curve describing the position in time of car 1 upward and that of car 2 to the right until they are tangent at $\left(t_{1}, \Delta x+S_{2}\right)$, and noticing that the curvature of the former is bigger.

2. If $t_{1}<t_{2}$, then a collision occurs if and only if equation $-\frac{1}{2} \Delta a t^{2}+\Delta v t+\Delta x=0$ has a solution in $\left[0, t_{1}\right]$, where $\Delta v \triangleq v_{2}-v_{1}$, and $\Delta a \triangleq a_{2}-a_{1}$. This condition can be shown to be equivalent to $\Delta v^{2}+2 \Delta x \Delta a>0$ and $\Delta v<0$.

Summing up, we get the overall condition:

$$
\Delta x \geq \max \left(0, \frac{v_{1}^{2}}{2 a_{1}}-\frac{v_{2}^{2}}{2 a_{2}}, \frac{\left(v_{2}-v_{1}\right)^{2}}{2\left(a_{1}-a_{2}\right)} 1_{S}\right),
$$

where $1_{S}$ is the indicator function of the set $S$ with $S \triangleq\left\{a_{1}>a_{2}, v_{1}>v_{2}, v_{2} / a_{2}>v_{1} / a_{1}\right\}$. Define the right hand side of $(5)$ to be $d_{\min }\left(a_{1}, a_{2}, v_{1}, v_{2}\right)$.

If there is a delay, say $\delta$, in the response of car 1 to the deceleration of car 2 , we simply modify the previous analysis by looking at the time instant $t=\delta$ car 1 begins braking. Assuming car 2 does not come to a stop up to time $\delta$ (which is quite realistic), we replace $\Delta x$ by $\Delta x(\delta)=\Delta x+\left(v_{2}-v_{1}\right) \delta-a_{2} \delta^{2} / 2$, and $v_{2}$ by $v_{2}(\delta)=v_{2}-a_{2} \delta$ in equation (5), thus obtaining:

$$
\Delta x(\delta) \geq \max \left(0, \frac{v_{1}^{2}}{2 a_{1}}-\frac{v_{2}(\delta)^{2}}{2 a_{2}}, \frac{\left(v_{2}(\delta)-v_{1}\right)^{2}}{2\left(a_{1}-a_{2}\right)} 1_{S(\delta)}\right)
$$

where $S(\delta) \triangleq\left\{a_{1}>a_{2}, v_{1}>v_{2}(\delta), v_{2}(\delta) / a_{2}>v_{1} / a_{1}\right\}$. Hence, in this case

$$
\begin{aligned}
d_{\min }= & \max \left(0, \frac{v_{1}^{2}}{2 a_{1}}-\frac{v_{2}(\delta)^{2}}{2 a_{2}}, \frac{\left(v_{2}(\delta)-v_{1}\right)^{2}}{2\left(a_{1}-a_{2}\right)} 1_{S(\delta)}\right) \\
& -\left(v_{2}-v_{1}\right) \delta+\frac{1}{2} a_{2} \delta^{2}
\end{aligned}
$$

i.e., $d_{\min }$ is also a function of $\delta$.

For an alternative treatment, see [8].

\section{PC with emergency braking}

In reality, the probability that the car in the lead will apply emergency braking is, although small, not zero. The frequency of emergency braking instances depends on factors such as traffic, road condition, weather, and the reliability of the cars ahead. To accommodate this into the model presented in Section 2, we make the following assumptions:

- Car 2 will execute emergence braking according to an exponentially distributed time interval $\tau$, independently of its motion;

- Once car 1 and car 2 decide to brake, they will keep braking at their maximal deceleration capability $a_{1}$ and $a_{2}$, respectively.

The choice of an exponentially distributed time to brake $\tau$ is justified by its memoryless property: given the fact that car 2 has not braked so far, the distribution of the time it takes from that time instant for car 2 to brake does not change. Besides these two assumptions, everything remains the same as in the previous sections. In particular, the velocity difference and the initial distance are respectively denoted with $\Delta v=v_{2}-v_{1}$ and $\Delta x$. The perturbation is a scaled 1-D BM $\sigma B_{t}$, attributed to the motion of car 2. For simplicity, we assume that when car 2 starts braking, car 1 brakes istantaneously $(\delta=0)$.

If car 2 brakes at time $t$, then car 1 is safe if and only if $\Delta x(t) \geq d_{\min }\left(a_{1}, a_{2}, v_{1}(t), v_{2}(t)\right)$. If car 2 has not braked so far, then the time $\tau$ it chooses to do so satisfies $P(\tau>t)=e^{-\lambda t}$, where $\lambda$ is the rate of the exponential distribution of $\tau$ satisfying $E[\tau]=1 / \lambda$. Let $A$ be the event that a collision occurs and $C$ the event that a collision occurs before time $\tau$. Let $v_{2}(\tau)$ be the velocity of car 2 at time $\tau$ and assume car 1 maintains its speed $v_{1}$ before time $\tau$. Then:

$A=C \cup\left\{\Delta x+\Delta v \tau+\sigma B_{\tau} \leq d_{\min }\left(a_{1}, a_{2}, v_{1}, v_{2}(\tau)\right)\right\}$.

There are various ways to estimate $v_{2}(\tau)$; for example, one can assume that $v_{2}(\tau)=v_{2}$, i.e., car 2 maintains its initial speed. Or we can use the estimated 
average velocity $v_{2}(\tau)=v_{2}+\sigma B_{\tau} / \tau$ whose statistical mean is the former. We adopt the former for simplicity and use $d_{\min }$ to denote the resulting safe distance $d_{\min }\left(a_{1}, a_{2}, v_{1}, v_{2}\right)$.

To make the perturbation a standard 1-D BM $B_{t}$, we again scale $\Delta x, \Delta v$ and $d_{\min }$ by a factor of $1 / \sigma$ and get

$$
\Delta x^{\prime} \triangleq \Delta x / \sigma, \quad \Delta v^{\prime} \triangleq \Delta v / \sigma, \quad d_{\min }^{\prime} \triangleq d_{\min } / \sigma .
$$

Furthermore, we take the alternative viewpoint that car 2 is at a fixed position $\Delta x^{\prime}$ while the motion of car 1 is $B_{t}^{\mu}$, the BM with drift $\mu=-\Delta v^{\prime}$. So now

$$
A=C \cup\left\{\Delta x^{\prime}-B_{\tau}^{\mu} \leq d_{\min }^{\prime}\right\}
$$

where $C=\left\{\sup _{0 \leq t \leq \tau} B_{t}^{\mu} \geq \Delta x^{\prime}\right\}$.

The following formulae can be found in [9] without proof. For their elementary proofs, refer to [10].

Lemma 2 Let $t$ be a fixed time and $x \geq 0$. The joint distribution of sup $_{0 \leq s \leq t} B_{s}^{\mu}$ and $B_{t}^{\mu}$ is of the form:

$$
\begin{aligned}
& P\left(\sup _{0 \leq s \leq t} B_{s}^{\mu} \geq x, B_{t}^{\mu}=y\right) \\
= & \frac{1}{\sqrt{2 \pi t}} \exp \left(\mu y-\mu^{2} t / 2-(|y-x|+x)^{2} / 2 t\right) .
\end{aligned}
$$

Lemma 3 The joint distribution of $\sup _{0 \leq s \leq \tau} B_{s}^{\mu}$ and $B_{\tau}^{\mu}$ is: $(y<x)$

$$
\begin{aligned}
& P\left(\sup _{0 \leq s \leq \tau} B_{s}^{\mu} \geq x, B_{\tau}^{\mu}=y\right) \\
= & \frac{\lambda}{\sqrt{2 \lambda+\mu^{2}}} \exp \left(\mu y+(y-2 x) \sqrt{2 \lambda+\mu^{2}}\right) .
\end{aligned}
$$

Lemma $4 B_{\tau}^{\mu}$ has probability density function:

$$
P\left(B_{\tau}^{\mu}=y\right)=\frac{\lambda}{\sqrt{2 \lambda+\mu^{2}}} \exp \left(\mu y-|y| \sqrt{2 \lambda+\mu^{2}}\right) .
$$

We are now in a position to calculate PC in the presence of emergency braking. Define $d^{\prime} \triangleq d_{\min }^{\prime}$ and $\beta \triangleq \sqrt{2 \lambda+\mu^{2}}$. By $(7)$

$$
\begin{aligned}
P(A) & =\int_{\Delta x^{\prime}-d^{\prime}}^{\infty} P\left(B_{\tau}^{\mu}=y\right) d y \\
& \quad+\int_{-\infty}^{\Delta x^{\prime}-d^{\prime}} P\left(\sup _{0 \leq s \leq \tau} B_{s}^{\mu} \geq \Delta x^{\prime}, B_{\tau}^{\mu}=y\right) d y \\
& =\int_{\Delta x^{\prime}-d^{\prime}}^{\infty} \frac{\lambda}{\beta} e^{\mu y-\beta|y|} d y+\int_{-\infty}^{\Delta x^{\prime}-d^{\prime}} \frac{\lambda}{\beta} e^{\mu y+\beta\left(y-2 \Delta x^{\prime}\right)} d y
\end{aligned}
$$

Using equation (6), we have

Theorem 2 The probability of collision with emergency braking, $P_{c e}(d, \Delta v, \Delta x)$, is:

$$
\begin{cases}\frac{1}{2 \beta}\left[\beta+\mu+(\beta-\mu) e^{-2 \beta d / \sigma}\right] e^{(\Delta x-d)(\mu-\beta) / \sigma}, & \text { if } \Delta x \geq d \\ 1-\frac{\lambda}{\beta(\beta+\mu)}\left(1-e^{-2 \beta \Delta x / \sigma}\right) e^{(\Delta x-d)(\mu+\beta) / \sigma}, & \text { if } \Delta x<d\end{cases}
$$

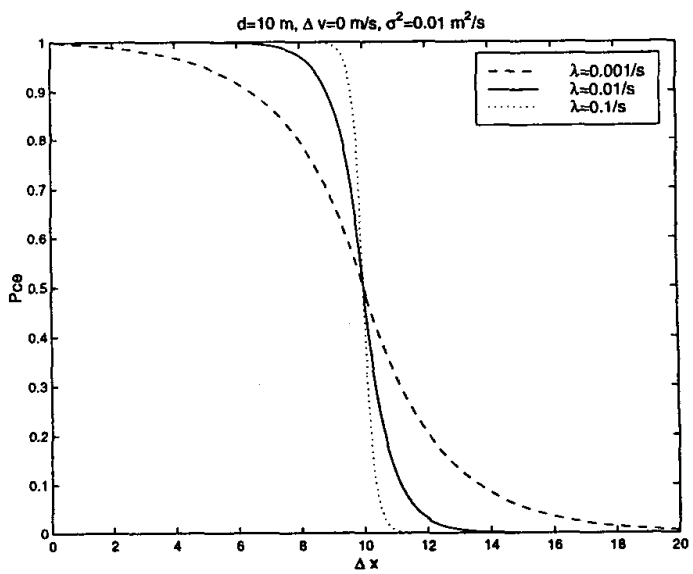

Figure 6: $P_{c e}$ for different values of $\lambda$.

where $d=d_{\min }\left(a_{1}, a_{2}, v_{1}, v_{2}\right), \mu=-\Delta v / \sigma$, and $\beta=$ $\sqrt{2 \lambda+\mu^{2}}$.

\section{Discussion and Future Directions}

In Figure 6, some plots of $P_{c e}$ as a function of $\Delta x$ are drawn for different values of $\lambda$, with $d=10 \mathrm{~m}$, $\sigma^{2}=0.01 \mathrm{~m}^{2} / \mathrm{s}$. Notice that as $\lambda \rightarrow \infty, P_{c e} \rightarrow 1_{(\Delta x<d)}$. This is expected since $\lambda \rightarrow \infty$ implies that with probability nearly 1 car 2 will brake almost instantly, while the distance between the two cars by then can not differ significantly from its initial value $\Delta x$. On the other hand, if $\lambda \rightarrow 0, P_{c e}$ will increase to 1 for any $\Delta x$ provided $\Delta v<0$. This is because when $\lambda$ is near $0, P_{c e}$ is approximately $P$ \{collision ever occurs , which is 1 for $\Delta v<0$. One simple way to get rid of this undesired "feature" is to put a lower bound on $\lambda$, that is, assume that emergency braking will eventually happen.

Figure 7 shows a comparison between the results of Theorem 2 and Theorem 1 . We set $\lambda=0.1 \mathrm{~s}^{-1}, \sigma=$ $0.01 \mathrm{~m}^{2} / \mathrm{s}$, and $t_{f}=10 \mathrm{~s}$ such that $E[\tau]=1 / \lambda=t_{f}$. In addition we set $d=0 \mathrm{~m}$, thus collision during emergency braking is ignored in the computation of $P_{c e}$. It can be seen that the two curves are close when $\Delta v$ is small. However as $\Delta v$ gets larger and larger, the discrepancy between them becomes more evident.

From the above discussion, we can infer that $\mathrm{PC}$ given by Theorem 2 is a compromise of two extreme cases: letting $\lambda \rightarrow \infty$, we get the conservative bound $d_{\text {min }}$, while letting $\lambda \rightarrow \lambda_{0}$ for some fixed $\lambda_{0}$, we get a bound which is approximately the one given by Theorem 1 with $t_{f}=1 / \lambda_{0}$ provided $d=0$.

Figure 8 shows $P_{c e}$ as a function of $\Delta x$ for different 


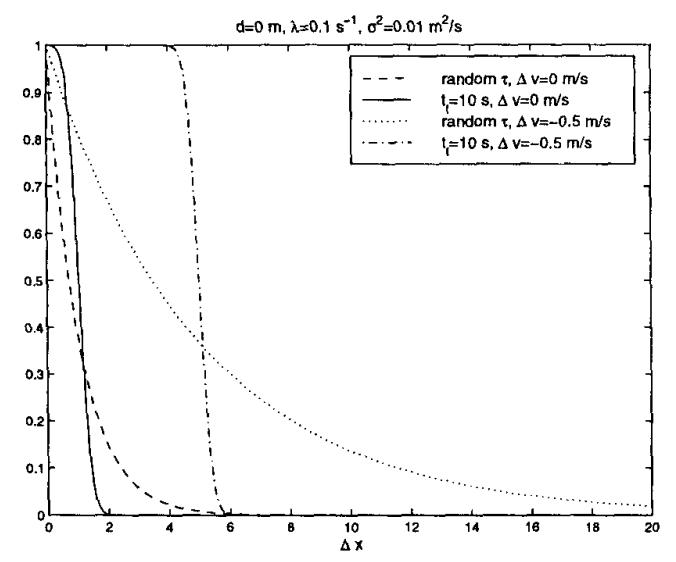

Figure 7: Deterministic vs. probabilistic stopping.

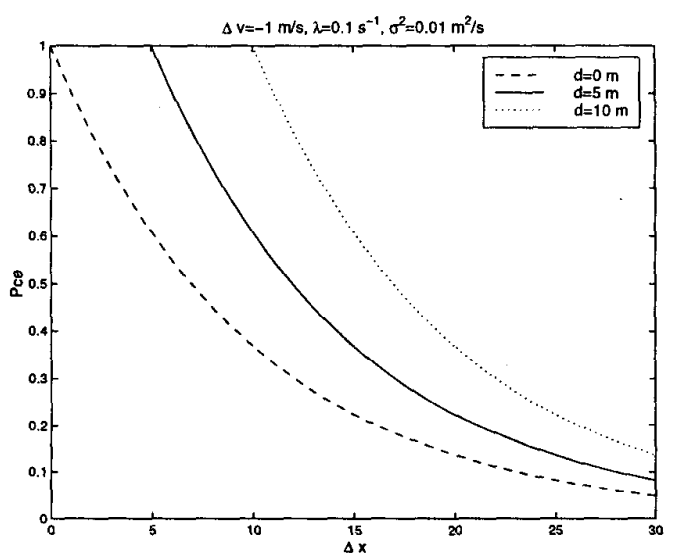

Figure 8: $P_{c e}$ for different values of $d$.

values of $d$. As the required safe distance $d$ increases, $P_{c e}$ also increases. In the case when the cars are approaching each other rather quickly, $P_{c e}$ is almost 1 if their distance is smaller than $d$.

Finally, some remarks about the choice of the power spectral density $\sigma^{2}$. Choosing different $\sigma$ will not alter the general shape of the curves, while larger $\sigma$ will in general lead to smoother decay of $P_{c e}$. Empirical data allowing an estimate of the value of $\sigma$ are not available yet, since real systems usually contain state feedback and are not open loop systems as assumed here. However, the necessity of having an accurate $\sigma$ is arguable since our main goal is to use $\mathrm{PC}$ as a guide in designing controllers. To this end, $\sigma$ can be used only as a parameter reflecting the "trust" each car has in its neighbors.

The advantage of having a closed form (and also smooth) expression for PC is that it can greatly facilitate the construction of controllers optimizing perfor- mance indices related to that expression. Furthermore, $\mathrm{PC}$ given in Theorem 1 and Theorem 2 can be used to determine the vehicles spacing guaranteeing a certain level of safety, in an attempt to achieve an "optimal" balance between safety and highway capacity. Work in these directions is currently underway.

\section{Appendix}

Refer to [10] for the proofs of these formulae.

Formula 1 Denote with $\beta \triangleq \sqrt{2 \lambda+\mu^{2}}$. Then

$$
\int_{0}^{\infty} \frac{\lambda e^{-\lambda t}}{\sqrt{2 \pi t}} e^{-(x-\mu t)^{2} / 2 t} d t=\frac{\lambda}{\beta} e^{\mu x-\beta|x|}
$$

Formula 2 Let $p(t)=\frac{a}{\sqrt{2 \pi t^{3}}} \exp \left(-\frac{(a+b t)^{2}}{2 t}\right)$. Then, for $a>0, b \neq 0$ :

$$
\int_{0}^{s} p(t) d t=Q\left(\frac{a+b s}{\sqrt{s}}\right)+e^{-2 a b} Q\left(\frac{a-b s}{\sqrt{s}}\right)
$$

\section{References}

[1] Bernt Oksendal, Stochastic differential equations, an introduction with application. Fifth edition, Springer-Verlag, 1998.

[2] P. Varaiya, "Smart cars on smart roads: problems of control", IEEE Transaction on Automatic Control, vol. AC-38, no. 2, 1993.

[3] Richard Durrett, Probability: theory and examples, Second edition, Duxbury Press, 1996.

[4] R.A. Paielli and H.Erzberger, "Conflict probability estimation for free flight", Journal of Guidance, Control and Dynamics, vol. 20, no. 3, pp. 588-596, 1997.

[5] J. Hu, J. Lygeros, M. Prandini, and S. Sastry, "Aircraft conflict prediction and resolution using brownian motion", in IEEE Conference on Decision and Control, Pheonix, AZ, 1999.

[6] S. Port and C. Stone, Brownian motion and classical potential theory, Academic Press, New York, 1978.

[7] Y.T. Yang and B.H. Tongue, "Intra-platoon collision behavior during emergency operations", Vehicle System Dynamics, vol. 23, no. 4, pp. 279-292, 1994.

[8] D. Godbole and J. Lygeros, "Safety and capacity analysis of automated highway systems", to appear in Transportation Research, Part C, 1999.

[9] A.N. Borodin, Handbook of Brownian motion, Birkhauser Verlag, Basel, Boston, 1996.

[10] J. Hu, J. Lygeros, M. Prandini, and S. Sastry, "Case study of collision probability", PATH technical report, UC Berkeley, to appear, 1999. 